\title{
Biodegradación de polietileno de baja densidad por acción de un consorcio microbiano aislado de un relleno sanitario, Lima, Perú
}

\author{
Biodegradation of low density polyethylene by the action of a microbial \\ consortium isolated from a landfill, Lima, Peru
}

\author{
Diego Uribe, Daniel Giraldo, Susana Gutiérrez y Fernando Merino
}

Laboratorio de Microbiología y Biotecnología Microbiana, Facultad de Ciencias Biológicas, Universidad Nacional Mayor de San Marcos. Apartado 110058, Lima 11, Perú. Email Diego Uribe: diegour24@yahoo.com

Trabajo presentado a la XVIII Reunión Científica del Instituto de Investigaciones en Ciencias BiolóInvestigaciones en Ciencias Bioló-
gicas Antonio Raimondi, "200 años del nacimiento de Charles Darwin y el 150 aniversario de la publicación de On the Origin of Species by Means of Natural Selection". Del 19 al 21 de agosto de 2009.

Publicado impreso: 20/10/2010 Publicado online: 29/09/2010

\section{Resumen}

En el presente trabajo describimos el aislamiento y la actividad de biodegradación de microorganismos sobre polietileno de baja densidad. Los microorganismos fueron aislados de materiales plásticos con evidencias de deterioro procedentes de un relleno sanitario de Lima. Las muestras fueron filtradas y preseleccionadas en medio de sales minerales a pH 5,5 y 7, para hongos y bacterias respectivamente. Se aislaron 6 cepas, identificadas como Pseudomonas sp. MP3a y MP3b, Penicillium sp. MP3a, Rhodotorula sp. MP3b, Hyalodendron sp. MP3c y una levadura no identificada. La acción degradativa del consorcio microbiano aislado fue evidenciada por variaciones en el espectro infrarrojo del polietileno con respecto al polímero sin tratamiento, observándose la reducción del índice de carbonilo $(83,89 \%$ a pH 7 y $4,08 \%$ a pH 5,5$)$ y de terminaciones con dobles enlaces $(19,77 \%$ a pH 7 y $6,47 \%$ a pH 5,5). Finalmente se determinó el porcentaje de peso perdido por el polietileno sometido a las cepas aisladas, observándose una disminución de $5,4 \%$ a pH 7 y $4,8 \%$ a pH5,5.

Palabras claves: Espectroscopia, Polietileno, Plásticos, Biodegradación, Polímeros.

\section{Abstract}

In this paper, we describe the isolation and biodegradation activity of microorganisms on low density polyethylene. The microorganisms were collected from plastic materials with evidence of deterioration from a landfill. The samples were filtered and selected in a mineral salts medium at $\mathrm{pH} 5.5$ and 7 for bacteria and fungi respectively. Six strains were isolated, identified as Pseudomonas sp. Hyalodendron sp., Penicillium sp. and Rhodotorula sp. Microbial activity was evidenced by changes in the infrared spectrum of polyethylene with respect to the polymer without treatment. Reduction of carbonyl index $(83.89 \%$ at $\mathrm{pH} 7$ and $4.08 \%$ at $\mathrm{pH} 5.5)$ and double bonds index $(19.77 \%$ at $\mathrm{pH} 7$ and $6.47 \%$ at $\mathrm{pH} 5.5)$ were observed. Finally we determined the percentage of weight lost by the polyethylene subjected to activity of the strains, with a decrease of $5.4 \%$ at $\mathrm{pH} 7$ and $4.8 \%$ at $\mathrm{pH} 5,5$.

Keywords: Spectroscopy, Polyethylene, Plastics, Biodegradation, Polymers.

\section{Introducción}

En la actualidad, los plásticos son productos muy utilizados y fabricados en grandes cantidades; sin embargo, debido a su difícil mineralización son uno de los contaminantes más importantes de los suelos y océanos (Allsopp et al. 2007).

Las investigaciones frente al problema de los plásticos, están dirigidas a la búsqueda de diferentes alternativas de reúso o degradación. Han sido producidos variantes de plásticos que contienen pro-oxidantes o polímeros biológicamente degradables, y que permiten su completa mineralización (Burgess-Cassler et al. 1991; Scott 1990; Johnson et al. 1993). En la actualidad, adquieren especial importancia las investigaciones de bacterias, actinomicetos y hongos (Lee et al. 1990) que biodegradan de manera óptima estos polímeros, o determinan las condiciones que favorecerían esta acción en el medio ambiente (Bonhommea et al. 2003; Orhan et al. 2004). Por otro lado, se investigan microorganismos capaces de sintetizar polímeros biodegradables para crear nuevos plásticos (Martins \& Marconato 2006), y microorganismos que producen enzimas extracelulares que alteran las propiedades físicas y químicas del polímero (Burgess-Cassler et al. 1991; Pometto et al. 1992; Iman y Gould 1990; Ishigaki et al. 2000).

El presente trabajo investiga muestras de material plástico deteriorado, en la búsqueda de microorganismos con capacidad biodegradativa sobre el polietileno de baja densidad (PEBD) y evalúa en condiciones controladas su actividad.

\section{Materiales y métodos}

Colecta.- Tres muestras de materiales plásticos con signos de deterioro fueron colectados entre los 15 y $20 \mathrm{~cm}$ de profundidad, fueron colectados de la plataforma No1 del Relleno Sanitario Portillo Grande en Lurín, Lima. Las muestras fueron trasladadas con material refrigerante al laboratorio para su procesamiento.

Enriquecimiento selectivo.- Las muestras fueron sumergidas en solución salina $(0,85 \% \mathrm{NaCl})$ agitando vigorosamente el contenido para resuspender los microorganismos adheridos al plástico; posteriormente fueron filtradas con Papel Whatman de diferente porosidad, finalmente se concentró en un filtro de membrana de $0,45 \mu \mathrm{m}$; el cual sirvió de inóculo para los medios de enriquecimiento a $\mathrm{pH}$ 7,0 para el aislamiento de bacterias y a $\mathrm{pH}$ 5,5 para la selección de hongos y levaduras.

Los microorganismos con capacidad biodegradativa fueron preseleccionados en un medio de sales minerales (MSM) (Bonhommea et al. 2003), compuesto de (g.L $\left.\mathrm{L}^{-1}\right) \mathrm{MgSO}_{4}\left(7 \mathrm{H}_{2} \mathrm{O}\right)$, 0,5 g; $\mathrm{KH}_{2} \mathrm{PO}_{4}, 0,5 \mathrm{~g} ; \mathrm{Na}_{2} \mathrm{HPO}_{4}\left(12 \mathrm{H}_{2} \mathrm{O}\right), 2,52 \mathrm{~g} ; \mathrm{NH}_{4} \mathrm{Cl}, 1 \mathrm{~g}$; $\mathrm{CaCl}_{2}, 0,002 \mathrm{~g} ; \mathrm{MnSO}_{4}(7 \mathrm{H} 2 \mathrm{O}), 0,007 \mathrm{~g} ; \mathrm{FeSO}_{4}(7 \mathrm{H} 2 \mathrm{O}), 0,001$ $\mathrm{g}_{\mathrm{y} \mathrm{ZnSO}_{4}}(7 \mathrm{H} 2 \mathrm{O}), 0,007 \mathrm{~g}$. Suplementado con extracto de levadura $0,02 \%$ y con $20 \mathrm{~g}$ de perlas de polietileno de baja densidad químicamente puro (única fuente de carbono), desinfectado en una solución de detergente e hipoclorito de sodio al 10\% (v/v), agua estéril temperada a $80^{\circ} \mathrm{C}$ y alcohol etílico de $70^{\circ}$.

Identificación.- Los cultivos se desarrollaron en recipientes con cierre hermético, ocupando menos del primer tercio del volumen total del frasco $(100 \mathrm{~mL})$; incubados durante 45 días a temperatura ambiente $\left(20^{\circ} \mathrm{C}\right)$. Se consideraron controles sin inoculación para ambas condiciones $(\mathrm{pH} 7,0$ y pH 5,5). Después de 45 días, $10 \mathrm{~mL}$ del cultivo de enriquecimiento fueron trasladados a un nuevo medio MSM + PEBD, pero sin extracto de levadura. Este cultivo fue desarrollado por 60 días. 


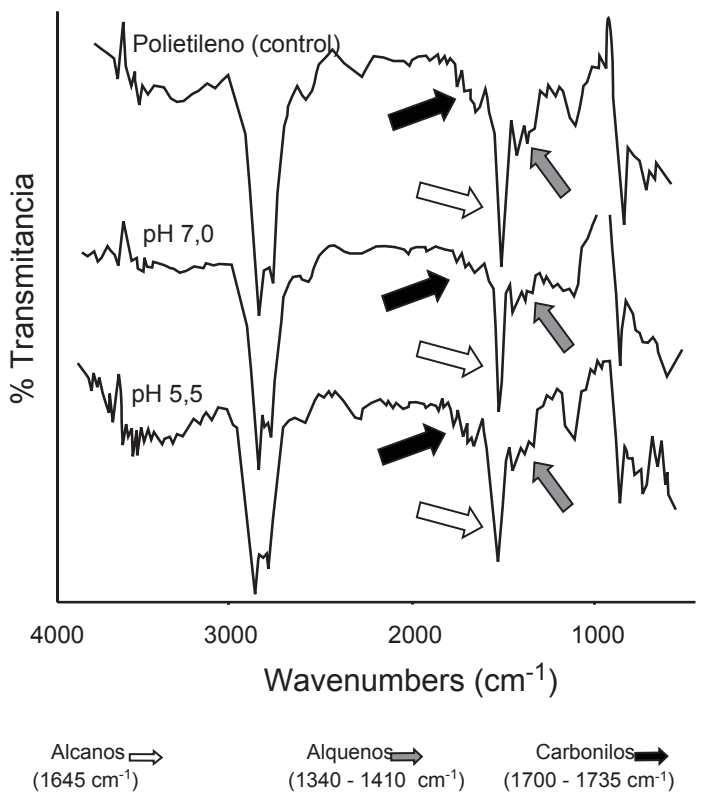

Figura 1. Análisis cualitativo de los espectros IR del PEBD de MP3, por superposición de gráficas.

Los microorganismos seleccionados en el enriquecimiento fueron sembrados por agotamiento en agar nutritivo, los cultivos a $\mathrm{pH} 7,0$, y en agar glucosado Sabouraud los correspondientes a pH 5,5. Los microorganismos obtenidos de cada aislamiento fueron conservados en agar TSA y ASG en plano inclinado, para los grupos microbianos encontrados.

Para la identificación de los géneros se realizaron pruebas bioquímicas para microorganismos Gram positivos (catalasa, tinción de esporas, motilidad, asimilación de carbohidratos) y API 20NE para Gram negativos aislados. Los hongos se identificaron mediante el desarrollo de microcultivos en ASG para el reconocimiento de estructuras reproductivas.

Análisis FTIR del polietileno de baja densidad.- Como un paso previo a la evaluación cuantitativa, se realizó un análisis por espectroscopia infra-roja de las perlas del PEBD utilizadas en uno de los cultivos de enriquecimiento, con el objetivo de constatar la existencia de un ataque microbiano por las cepas durante el proceso de pre-selección. Se utilizó un equipo FTIR (Fourier Transform Infrared Spectroscopy) donde se analizó el PEBD para obtener los grupos funcionales característicos y los índices de reducción de $\mathrm{C}-\mathrm{O}$ y $\mathrm{C}=\mathrm{C}$ que denotan efectos de biodegradación sobre el polímero.

Prueba cuantitativa de degradación de PEBD.- La prueba cuantitativa de la capacidad biodegradativa consistió en determi-
Tabla 1. Identificación de las cepas obtenidas en el aislamiento del consorcio MP3.

\begin{tabular}{ccc}
\hline \multirow{2}{*}{$\begin{array}{c}\text { Aislamiento } \\
\text { a pH 7,0 }\end{array}$} & $\begin{array}{c}\text { Cepas } \\
\text { aisladas }\end{array}$ & Microorganismo identificado \\
\cline { 2 - 3 } & $\mathrm{MP3a}$ & Pseudomonas sp. \\
& $\mathrm{MP3b}$ & Pseudomonas sp. \\
\hline \multirow{3}{*}{ Aislamiento } & $\mathrm{MP3a}$ & Penicillium $\mathrm{sp}$. \\
a pH 5,5 & $\mathrm{MP3b}$ & Rhodotorula $\mathrm{sp}$. \\
& $\mathrm{MP3c}$ & Hyalodendron sp. \\
& $\mathrm{MP4d}$ & Levadura no identificada \\
\hline
\end{tabular}

nar la cantidad de peso perdido en un periodo de incubación de 2 meses, de una lámina de PEBD previamente pesada.

Para esto se realizaron suspensiones de células en solución salina, compatibles al tubo 2 de McFarland, con las cepas aisladas correspondientes a cada consorcio, las cuales se inocularon en medio MSM $(100 \mathrm{~mL})$ con discos de PEBD previamente esterilizados y pesados en una balanza analítica $( \pm 0,001 \mathrm{~g})$. Las condiciones de incubación fueron similares a las mencionadas, durante 60 días. Al final de la incubación se desinfectó el PEBD para eliminar el biofilm generado en la superficie, se pesaron los discos de plástico y se determinó el porcentaje de peso perdido. El pesado se realizó por triplicado.

\section{Resultados y discusión}

Durante los últimos 2 meses de incubación, se observó en la muestra número 3 (MP3) el desarrollo de micelas sobre la superficie de las perlas del PEBD y la aparición de películas muy tenues, como evidencia de un posible ataque microbiano.

Se aislaron dos cepas en el cultivo a $\mathrm{pH}$ 7,0 y 4 cepas a $\mathrm{pH}$ 5,5 del cultivo desarrollado para MP3 (Tabla 1).

El análisis cualitativo del FTIR de las perlas de PEBD de los cultivos $\mathrm{MP} 3$, denotó variaciones en los picos correspondientes a los grupos funcionales presentes en el polietileno (polímero de etileno), los cuales son de naturaleza invariante (Fig. 1).

Los espectros mostraron variaciones significativas en los grupos C-O (control 1726,17 $\mathrm{cm}^{-1}$; $\mathrm{pH} 5,5,1727,97 \mathrm{~cm}^{-1} ; \mathrm{pH} 7,0$, $\left.1721,10 \mathrm{~cm}^{-1}\right)$ y $\mathrm{C}=\mathrm{C}$ (control $1371,77 \mathrm{~cm}^{-1} ; \mathrm{pH} 5,5,1371,05$ $\mathrm{cm}^{-1}$; $\mathrm{pH} 7,0,1371,85 \mathrm{~cm}^{-1}$ ) (Figs. 2, 3 y 4). Este cultivo fue elegido para esta prueba porque mostró evidencias de actividad microbiana por la aparición de micelas y pequeñas biopelículas en la superficie. El FTIR permitió evidenciar una reducción del $83,89 \%$ de la fuente de carbono correspondiente al grupo funcional C-O para $\mathrm{pH} 7,0$ y de la misma forma para $\mathrm{C}=\mathrm{C}$ con un índice de reducción de 19,77\% a pH 5,5 (Tabla 2).

Estos datos fueron obtenidos estableciendo una relación entre, la absorbancia del pico de los grupos funcionales $\mathrm{C}-\mathrm{O}$ (cercano

Tabla 2. Reducción del índice del grupo funcional carbonilo y del índice de terminaciones con dobles, durante el periodo de enriquecimiento de la muestra de plástico 3 .

\begin{tabular}{|c|c|c|c|}
\hline & Ac-o / A cH2 & $\%$ de Carbonilo & Reducción del índice de Carbonilo \\
\hline PEBD sin tratamiento & $0,0132 / 0,2441=0,0540$ & $100 \%$ & - \\
\hline PEBD + MP3* pH 5,5 & $0,0123 / 0,2373=0,0518$ & $95,92 \%$ & $4,08 \%$ \\
\hline \multirow[t]{2}{*}{ PEBD + MP3* pH 7,0 } & $0,0021 / 0,2403=0,0087$ & $16,11 \%$ & $83,89 \%$ \\
\hline & $\mathrm{Ac}=\mathrm{c} / \mathrm{A} \mathrm{CH} 2$ & $\%$ de $\mathrm{C}=\mathrm{C}$ & Reducción del índice de $C=C$ \\
\hline PEBD sin tratamiento & $0,0861 / 0,2441=0,0540$ & $100 \%$ & - \\
\hline PEBD + MP3* pH 5,5 & $0,0783 / 0,2373=0,0518$ & $93,53 \%$ & $6,47 \%$ \\
\hline PEBD + MP3* pH 7,0 & $0,0680 / 0,2403=0,0087$ & $80,23 \%$ & $19,77 \%$ \\
\hline
\end{tabular}




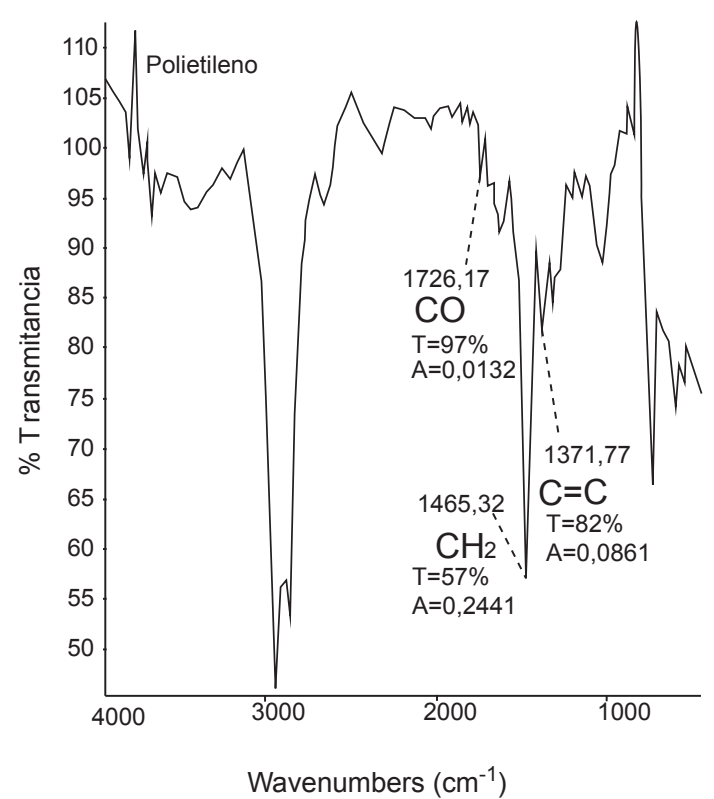

Figura 2. Análisis FTIR del PEBD sin inoculación, utilizado como patrón inicial.

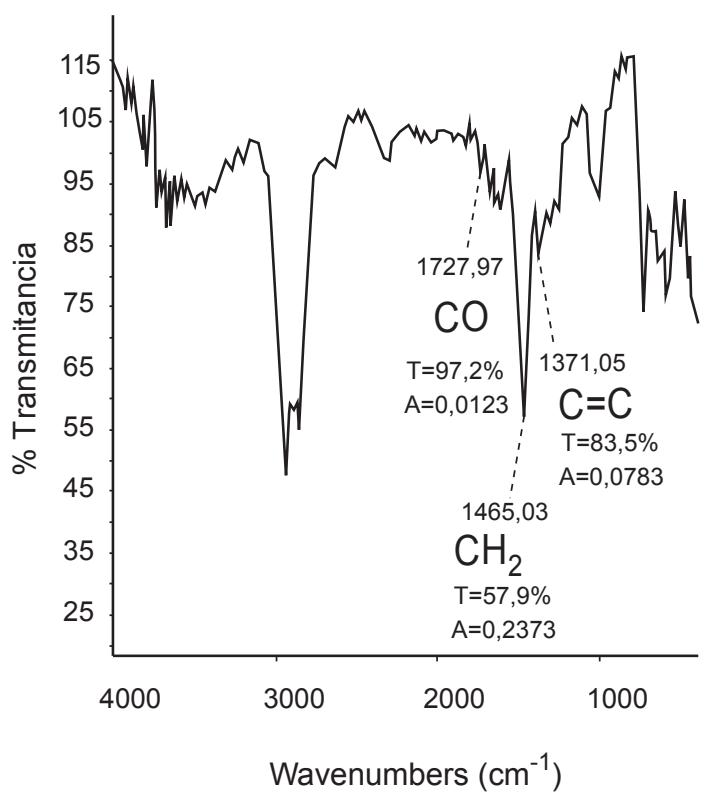

Figura 3. Análisis FTIR del PEBD después de 2 meses incubación con el consorcio MP3 a pH 5,5.

a $\left.1700-1735 \mathrm{~cm}^{-1}\right)$ y $\mathrm{C}=\mathrm{C}$ (cercano a $\left.1340-1410 \mathrm{~cm}^{-1}\right)$, y la absorbancia del pico de $\mathrm{CH}_{2}$ (cercano a 1465).

Se obtuvo una reducción de $5,4 \%$ del peso total de polietileno bajo la acción del consorcio conformado solo por bacterias $(\mathrm{pH} 7,0)$. El porcentaje de peso perdido obtenido mediante el empleo de las levaduras y los hongos aislados fue de 4,8\% (pH 5,5 ), que si bien es menor, es un resultado significativo para las condiciones en las que se desarrolló la prueba.

Las pruebas realizadas utilizaron una única fuente de carbono durante todo el periodo de incubación, principal característica de toda prueba de biodegradación. Esto se aplicó desde la pre-selección de las cepas para realizar el aislamiento. Para el primer cultivo de enriquecimiento decidimos emplear además de la fuente de carbono, un porcentaje muy pequeño $(0,02 \%)$ de extracto de levadura en el medio; ya que anteriormente fue probado con buenos resultados, aunque otros autores consideran

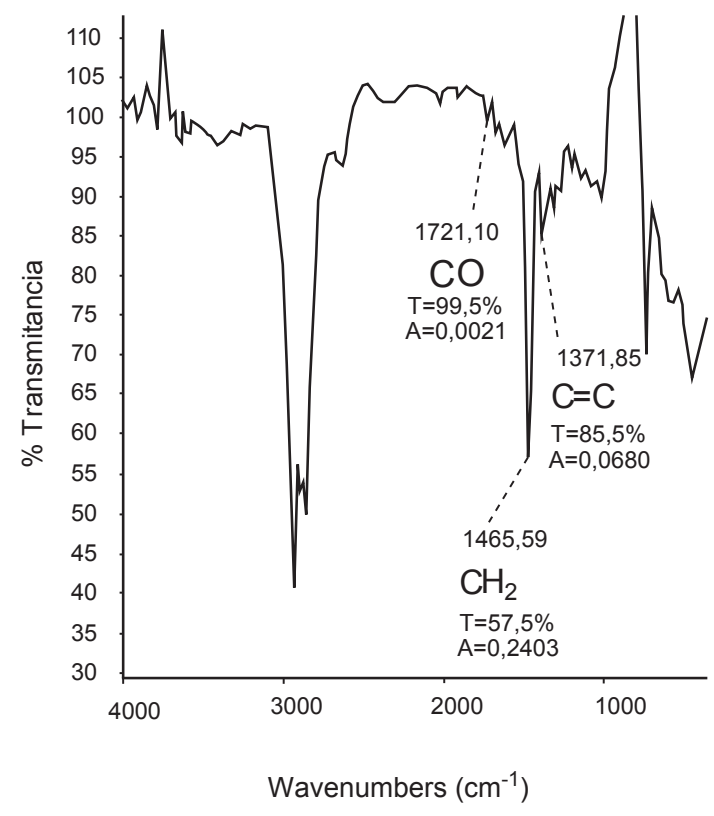

Figura 4. Análisis FTIR del PEBD después de 2 meses incubación con el consorcio MP3 a pH 7,0.

el uso de glucosa u otra fuente de carbono, que es suprimida del cultivo mediante pasajes sucesivos en determinados tiempos. Ante esto, inferimos que el empleo de un precursor de crecimiento en mínimas concentraciones ayudaría a "proponer" el desarrollo de un crecimiento moderado de los microorganismos presentes en la muestra, para que luego estos sean recuperados eficazmente en un posterior periodo de selección con la fuente de carbono en prueba, en este caso el polietileno de baja densidad.

Durante la segunda etapa de enriquecimiento, en el cual se empleo sólo MSM + PEBD, se pudo observar el desarrollo de micelas y una biopelícula formada en la superficie de las perlas del polímero, lo cual resaltó la importancia de esta muestra para el posterior aislamiento de sus consorcios. El desarrollo de biopelículas o micelas en la superfice del polietileno indicaría la acción deteriorante o degradativa de microorganismos.

El FTIR corresponde a una alternativa rápida y de costo moderado para evaluar la acción degradativa de algún agente sobre los polímeros sintéticos (Lucas et al. 2008), mediante la cuantificación de la reducción de los índices de grupos funcionales característicos del PEBD. El espectro infra-rojo (IR) se analiza separando dos zonas referenciales; la primera es llamada la región de los grupos funcionales que va de $1200 \mathrm{a} 3600 \mathrm{~cm}^{-1} \mathrm{y}$ la segunda denominada región de huella digital que se despliega desde $600 \mathrm{a} 1200 \mathrm{~cm}^{-1}$; esta última es una zona muy específica, donde los picos son invariantes para un polímero, a menos que este haya sufrido el efecto de algún agente químico, físico o biológico (Gulmine 2002). Es por esto que, por una simple superposición de los espectros podemos observar el efecto de los microorganismos sobre el polietileno tanto a $\mathrm{pH} 7,0$ y pH 5,5. El espectro IR denota cuantitativamente la reducción de los índices de carbonilo (C-O) y de las terminaciones con doble enlace $(\mathrm{C}=\mathrm{C})$. Durante el proceso de degradación, el grupo funcional carbonilo es liberado por acción de la luz UV y rápidamente asimilado como fuente de energía por los microorganismos, que a su vez podría permitir la mayor degradación de la estructura principal de la molécula del polietileno, también compuesta por cadenas de enlaces dobles. Es por esto que la reducción del índice de carbonilo obtenida en los cultivos a pH 7,0 guarda relación 
con la disminución del índice de terminaciones $\mathrm{C}=\mathrm{C}$, aunque en esto también está involucrado el tipo de microorganismos presentes en el cultivo.

Los géneros identificados en los consorcios del cultivo para MP3, son microorganismos mencionados en trabajos relacionados con biodegradación y biodeterioro de plásticos; así tenemos a diferentes especies Pseudomonas, que son capaces de ejercer actividad degradativa sobre polímeros como el poliuretano (Howard 2002) y el cloruro de polivinilo, entre otras moléculas, como el polietilen-glicol (Obradors \& Aguilar 1991), que también son presa de la versatilidad metabólica de esta cepa (Wasserbauer et al. 1990). Diferentes especies de Bacillus son capaces de producir una exoenzima que afecta al acetato de celulosa, material empleado para los revelados de Rayos X en medicina (Ishigaki et al. 2000). Especies de Penicillium muestran su actividad degradativa sobre polietileno en asociación con Bacillus sp. (Seneviratne et al. 2006). También han sido reportado biodeterioro ejercido por algas, y otras especies de microorganismos como Sphingomonas sp., Arthrobacter sp. (Imam \& Gould 1990), Streptomyces sp. (Lee et al. 1990), Brevibacillus sp. (Hadad et al. 2005) y Flavobacterium sp. (Koutny 2009). A esta lista, nuestro trabajo reporta Hyalodendron sp.

La actividad microbiana sobre los plásticos está dada por una acción enzimática, muchos autores proponen que la misma enzima iniciadora de la degradación de hidrocarburos (alcano monoxigenasa) es la responsable del ataque microbiano sobre la superficie de los polímeros sintéticos (Seneviratne 2006).

Cabe recalcar que la recuperación de una cepa en un consorcio de degradación, no necesariamente indica que esta es capaz de mineralizar por sí sola el polímero en su plenitud. Se han realizado estudios que indican que la presencia de hongos en este tipo de consorcios, genera la posibilidad de una duda en cuanto a su capacidad degradativa; y es que son tan versátiles bioquímicamente, que podrían estar tomando como fuente de carbono, los productos de degradación de las demás cepas integrantes del consorcio, por lo cual, sería importante la elaboración de pruebas de biodegradación individualizadas para cada microorganismo encontrado.

\section{Agradecimientos}

Los autores agradecemos al Dr. Pedro Castellanos, por la ayuda en la identificación de los hongos.

\section{Literatura citada}

Allsopp M., A. Walters, D. Santillo, et al. 2007. Contaminación por plásticos en los océanos del mundo. GreenPeace.< http://www.greenpeace.org/raw/content/espana/reports/ contaminaci-n-por-plasticos-en.pdf $>$ (access: 16/01/2010)

Bonhommea S., A. Cuerb, A.M. Delortb, et al. 2003 Environmental biodegradation of polyethylene. Polymer Degradation and Stability 81:441-452.
Burgess-Cassler A., S.H. Imam \& J.M. Gould 1991. High-Molecular-Weight Amylase Activities from Bacteria Degrading Starch-Plastic Films. Applied And Environmental Microbiology 57:612-614.

Gulmine J.V., P.R. Janissek, H.M. Heise, et al. 2002. Polyethylene characterization by FTIR. Polymer Testing $21: 557-563$.

Hadad D., S. Geresh \& A. Sivan 2005. Biodegradation of polyethylene by the thermophilic bacterium Brevibacillus borstelensis. Journal of Applied Microbiology 98: 1093-1100.

Howard G.T. 2002. Biodegradation of polyurethane: a review. International Biodeterioration \& Biodegradation 49: 245 - 252.

Imam S.H. \& Gould J.M. 1990. Adhesion of an Amylolytic Arthrobacter sp. To Starch-Containing Plastic Films. Applied and Environmental Microbiology 56: 872-876

Ishigaki T., W. Sugano, M. Ike, et al. 2000. Enzymatic degradation of cellulose acetate plastic by novel degrading bacterium Bacillus sp. S2055. Journal of Bioscience and Bioengineering 90(4): 400-405.

Johnson K. E., A.L. Pometto III \& Z.L. Nikolov 1993. Degradation of degradable starch-polyethylene plastics in a compost environment. Applied And Environmental Microbiology 59: 1155-1161.

Koutny M., P. Amato, M. Muchova, et al. 2009. Soil bacterial strains able to grow on the surface of oxidized polyethylene film containing prooxidant additives. International Biodeterioration \& Biodegradation 63: 354-357.

Lee B., A.L. Pometto III, A. Fratzke, et al. 1990. Biodegradation of degradable plastic polyethylene by Phanerochaete and Streptomyces species. Applied And Environmental Microbiology 57: 678-685.

Lucas N., C. Bienaime, C. Belloy, et al. 2008. Polymer biodegradation: Mechanisms and estimation techniques. Chemosphere 73: 429-442

Martins S. M. \& J.C. Marconato 2006. Polímeros Biodegradáveis - uma Solução Parcial Para Diminuir a Quantidade Dos Resíduos Plásticos. Quim. Nova 29(4): 811-816.

Obradors N. \& J. Aguilar 1991. Efficient Biodegradation of HighMolecular-Weight Polyethylene Glycols by Pure Cultures of Pseudomonas stutzeri. Applied And Environmental Microbiology 57 (8): 2383-2388

Orhan Y., J. Hrenović \& H. Büyükgüngör. 2004. Biodegradation of Plastic Compost bags under controlled soil conditions. Acta Chim. Slov. 51: 579-588.

Pometto III A.L., B. Lee \& K.E. Johnson 1992. Production of an Extracellular Polyethylene-Degrading Enzyme(s)by Streptomyces Species. Applied And Environmental Microbiology 58:731-733.

Scott G. 1990. Photo-biodegradable Plastics: Their Role in the Protection of the Environment. Polymer Degradation and Stability 29: 135-154.

Seneviratne G., N. S. Tennakoon, M. L. M. A. W. Weerasekara et al 2006. Polyethylene biodegradation by a developed Penicillium-Bacillus biofilm. Current Science 90 (1): 20-21.

Wasserbauer R., M. Beranovti \& D. Vancurovk 1990.Biodegradation of polyethylene soils by bacterial and liver homogenates. Biomaterials 11:36-40 